\title{
Anaesthetic effects of tiletamine-zolazepam-xylazine-tramadol combination in cats undergoing surgical sterilization
}

\author{
Lin $\mathrm{Li}^{1 \mathrm{a}}$, Jing Dong ${ }^{1 \mathrm{a}}$, Jianbin $\mathrm{He}^{1}$, Jixian $\mathrm{Cui}^{2}$, Xuewu $\mathrm{Yu}^{2}$, Dezhan $\mathrm{Tan}^{3}$, Honggang $\mathrm{Fan}^{4}$ \\ ${ }^{1}$ Shenyang Agricultural University, College of Animal Husbandry and Veterinary Medicine, Shenyang, China \\ ${ }^{2}$ Institute of Animal Medical Research, Animal Disease Prevention and Control Center of Liaoning Province, \\ Shenyang, China \\ ${ }^{3}$ Shaoyang Vocational and Technical College, Shaoyang, China \\ ${ }^{4}$ Northeast Agricultural University, College of Veterinary Medicine, Harbin, China

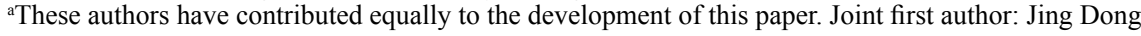

Received July 23, 2014

Accepted January 14, 2015

\begin{abstract}
This study was conducted to evaluate the anaesthetic and physiological effects of tiletaminezolazepam-xylazine-tramadol (TZXT) combination in cats undergoing surgical sterilization. A total of $20,13.3 \pm 2.5$ months old, with a body weight of $2.4 \pm 0.2 \mathrm{~kg}$, male cats were used in the experiment. Cats were randomly assigned into the anaesthesia group (group A) or to the surgery group (group S). The tiletamine-zolazepam-xylazine-tramadol combination was administered with an intended dose of $2.4 \mathrm{mg} \cdot \mathrm{kg}^{-1}$ tiletamine-zolazepam (TZ), $0.36 \mathrm{mg} \cdot \mathrm{kg}^{-1}$ xylazine (X), and $0.8 \mathrm{mg} \cdot \mathrm{kg}^{-1}$ tramadol (T) into the lateral femoral muscle. Physiological indicators including rectal temperature (RT), heart rate (HR), respiration rate (RR), haemoglobin saturation by oxygen $\left(\mathrm{SpO}_{2}\right)$, mean arterial pressure (MAP), systolic arterial pressure (SAP), and diastolic arterial pressure (DAP) were collected after baseline values (time 0). Cats were immediately given the intramuscular injection drug combination. Physiological indicators were recorded before injection of this combination and at time 5, 10, 15, 20,30, 40, 50, 60, 80, 100, and $120 \mathrm{~min}$ after administration of the tiletamine-zolazepam-xylazine-tramadol combination. In group $\mathrm{S}$, castrations were performed using a closed procedure. Vomiting, excitement, apnoea, and abnormal behaviour were not observed in any cat during anaesthesia. All changes in indicators were within cardiorespiratory acceptable limits in both groups. The tiletamine-zolazepam-xylazine-tramadol combination produced satisfactory anaesthesia in cats and it will be useful not only in induction of anaesthesia but also for surgery of short duration.
\end{abstract}

Castration, immobilization, heart rate, respiration rate, mean arterial pressure

Cats have always been an anaesthetic challenge for their unique temperament, dramatic stress response, relatively small size, and idiosyncratic drug metabolism. The demand for surgical procedures and sophisticated medical approach has added to the complexity of cat anaesthesia.

Tiletamine-zolazepam (Telazol, $50 \mathrm{mg} \cdot \mathrm{ml}^{-1}$ of tiletamine and zolazepam in a $5 \mathrm{ml}$ vial) and ketamine are the only short-acting injectable anaesthetics that may be administered intramuscularly and are approved for cats in the United States. But ketamine has been limited in its application as it is a controlled drug. Tiletamine-zolazepam administered intramuscularly has been reported to induce muscular relaxation and profound analgesia in cats, dogs, and a number of exotic species (Hellyer et al. 1988; Lin et al. 1993; De gerfeld 2005). Tiletaminezolazepam combination produces good restraint but can be associated with prolonged recoveries (Bednarski et al. 1989). Xylazine is an $\alpha_{2}$-adrenoreceptor agonist and has been combined with tiletamine-zolazepam to increase its analgesic and anaesthetic effects and to reduce the dose of tiletamine-zolazepam required to induce satisfactory anaesthesia. The drug has been approved by the US Food and Drug Administration for use in cats. However, its use is associated with adverse effects including unwanted persistence of pharmacological effects and hypertension

Address for correspondence:

Prof. Honggang Fan

College of Veterinary Medicine

Northeast Agricultural University

Harbin 150030, China
Phone: 86-0451-55190470

Fax: 86-0451-55190470

E-mail: fanhonggang2002@163.com

http://actavet.vfu.cz/ 
(James et al. 1999; Lemke 2004). Xylazine binds to membrane-associated receptors on presynaptic and postsynaptic nerves. The presynaptic receptors appear to modulate the release of noradrenaline, resulting in sedation, decreased locomotor activity and the suppression of conditioned responses. The $\alpha$-receptors also play a role in the modulation of degree of alertness, blood pressure, pupillary diameter, gastrointestinal electrolyte absorption and blood glucose concentration (Doherty 1988). However, complete immobility requires the addition of other anaesthesia agents such as tiletamine-zolazepam. But adverse effects such as vomiting, salivation and agitation could be observed during induction sometimes (James et al. 1999).

Tramadol has been used clinically for the last two decades to treat moderate to moderately severe pain in humans through interactions with serotonin, opioid, and adrenergic receptors. Animals with liver or kidney disease need lower doses of tramadol (Engelhardt et al. 2003; Safarinejad and Hosseini 2006) but tramadol has not been used in pets for very long time. We know little about possible long-term toxicity when it is used in cats. Perhaps it will turn out to be an effective alternative to NSAIDs (Non-Steroid Anti-Inflammatory Drugs). Tramadol is a centrally acting analgesic with weak opioid agonist properties, and effects on serotonergic and noradrenergic neurotransmission (Lehmann 1997). It has been proven effective in both experimental and clinical pain without causing serious respiratory or cardiovascular adverse effects. Tramadol has been widely used in horses and goats (Witte et al. 2001; Shilo et al. 2008; Sousa et al. 2008).

Based on this background and our clinical experience we developed an anaesthetic combination of tiletamine-zolazepam-xylazine-tramadol (TZXT) for intramuscular administration in cats. In a previous study, the anaesthetic combination showed a good anaesthetic effect in cats (Lin et al. 2012), but no information has been published to our knowledge on the effects of this drug combination in cats undergoing surgery. The aim of this study was to evaluate the anaesthetic and cardiorespiratory effects of TZXT in cats undergoing surgical sterilization.

\section{Materials and Methods}

A total of 20 male cats were randomly assigned into the anaesthesia group (group A), and the surgery group (group S). Cats were admitted to the study only if they were an adult, based on the presence of permanent canine teeth and physical appearance, and only if they required routine sterilization procedures.

Age and body weight of animals expressed as mean $\pm \mathrm{SD}$ were $13.3 \pm 2.5$ months, and $2.4 \pm 0.2 \mathrm{~kg}$, respectively. Animals used in this experiment were approved by the Animal Care and Use Committee of the Shenyang Agricultural University. All cats were determined to be in a good physical condition based on physical examination and complete blood count.

Feed was withheld for $12 \mathrm{~h}$ prior to the start of the experiment, except for water. Each cat was allowed to acclimate to room temperature at $25^{\circ} \mathrm{C}$ for at least $30 \mathrm{~min}$ before the experiment started. After that each cat was caught in a net and weighed. Baseline physiological indicators including rectal temperature (RT), heart rate $(\mathrm{HR})$, respiration rate $(\mathrm{RR})$, haemoglobin saturation by oxygen $(\mathrm{SpO} 2)$, mean arterial pressure (MAP), systolic arterial pressure (SAP), and diastolic arterial pressure (DAP) were measured by noninvasive monitor (DatexOhmedaS/5TM, Datex-Ohmeda Division Instrumentarium Corp, Helsinki, Finland) before drug administration. Blood pressure was obtained by placing a cuff circumferentially around the left antebrachium of the animal, with the cuff width being approximately $40 \%$ of the total circumference of the limb. The heart rate was determined by counting heart beats for $1 \mathrm{~min}$ using a stethoscope placed at the lower left lateral thoracic wall and RR was counted from thoracic excursions for $1 \mathrm{~min}$.

Tiletamine-zolazepam-xylazine-tramadol was administered by using a hand held syringe with an intended dose of $2.4 \mathrm{mg} \cdot \mathrm{kg}^{-1}$ tiletamine-zolazepam (Zoletil ${ }^{\circledR} 100$, Virbac corporation, Carros, France), $0.36 \mathrm{mg} \cdot \mathrm{kg}^{-1}$ xylazine (Rompun, Bayer, Leverkusen, Germany), and $0.8 \mathrm{mg} \cdot \mathrm{kg}^{-1}$ tramadol (Tramal ${ }^{\circledR} 100$; Grunenthal $\mathrm{GmbH}$, Aachen, Germany) into the lateral femoral muscle. After baseline values (time 0) for physiological indicators were collected, cats were immediately given intramuscular injection of the drug combination; physiological indicators were then recorded at 5, 10, 15, 20, 30, 40, 50, 60, 80, 100, and 120 min. After immobilization and antinociception with no response to the $20 \mathrm{G}$ pin-prick in the perineal region, they were placed in dorsal recumbency position on an operating table and an ophthalmic ointment was put into the eyes to prevent corneal drying. In group S, the urinary bladder of cats was manually expressed. The surgical site was prepared using standard aseptic technique. Castrations were performed using a closed procedure. In 
group A, cats did not undergo surgery and were used for collecting data on physiological indicators by the method of a group S.

Induction time was considered as the time from injection to complete immobilization. Complete immobilization was defined as a lack of response to handling. Anaesthesia time was considered as the time interval between complete immobilization and the first attempt made by the animal to lift its head a few centimetres above the ground.

Cardiorespiratory indicators were analysed by means of ANOVA for repeated measures to evaluate changes within each group and between two different treatments. All statistical analyses were performed using the SPSS version 13.0 software (SPSS Incorporation, Chicago, Illinois). A probability level of 5\% $(P<0.05)$ was considered significant. All values are reported as mean \pm standard deviation.

\section{Results}

After TZXT administration, all animals showed signs of dissociative anaesthesia characterised by mydriasis and licking of the nose. Vomiting, excitement, apnoea, and abnormal behaviour were not observed in any cat. In both groups the time of anaesthesia induction was $2.19 \pm 0.88 \mathrm{~min}$. The combination of anaesthetics resulted in the anaesthesia lasting about $52.42 \pm 11.79 \mathrm{~min}$ and muscular relaxation in the anaesthetic stage. In addition, the surgical procedure required $12 \pm 7 \mathrm{~min}$ to be completed in cats. Recovery was characterised by slow movements of the eyes, twitching of the ears, and attempts to lift the head. All cats were calm during the recovery period. Vomiting, excitement, apnoea, and abnormal behaviour were not observed in any cat. All animals were calmer and the features such as ataxia and uncontrolled manner were rarely observed in group A and the animals were able to stand and walk after one or two attempts without ataxia in group S during recovery.

Cardiorespiratory indicators are shown in Table 1. There was no significant difference in these indicators between two groups except for RT. There were similar change trends in both groups, RT in group S decreased more markedly and had a significant difference compared to group A at $20-50$ min after injection $(P<0.05)$, but all these changes in indicators were within cardiorespiratory acceptable limits.

\section{Discussion}

This study showed that in cats, addition of tramadol to tiletamine-zolazepam-xylazine improved the quality of anaesthetic induction and increased the analgesic effect without adversely affecting the cardiopulmonary indicators.

Tiletamine-zolazepam-xylazine-tramadol combination produced a complete immobilization in cats in our study. Alexis et al. (2004) also reported that tiletaminezolazepam $\left(2.8-5.6 \mathrm{mg} \cdot \mathrm{kg}^{-1}\right)$ and xylazine $\left(1.1-2.2 \mathrm{mg} \cdot \mathrm{kg}^{-1}\right)$ anaesthesia resulted in an induction period of $4 \pm 1 \mathrm{~min}$ and recovery time $108 \pm 24 \mathrm{~min}$ in cat. The doses of tiletamine-zolazepam and xylazine used in our study were much lower. Tiletaminezolazepam-xylazine-tramadol combination had a quicker induction and better recovery when compared with tiletamine-zolazepam-xylazine combination, most likely because of the prolonged sedative effect of tramadol, the addition of tramadol increased significantly the duration of antinociception.

Rectal temperature in the surgery group showed a significant difference compared to the anaesthesia group at 20-50 min and decreased more markedly. The reason is that laparotomy and saline flush caused loss of body heat during this period. The change trends of $\mathrm{HR}, \mathrm{RR}, \mathrm{SpO}, \mathrm{SBP}, \mathrm{DBP}$, and MAP in the surgery group were similar as in the anaesthesia group. There was no significant difference between the two groups. Muscle relaxation was good with no resistance when opening the mouth and no complications were encountered during surgical procedures. It indicates that TZXT will be useful not only in induction of anaesthesia but also for surgeries of short duration. 


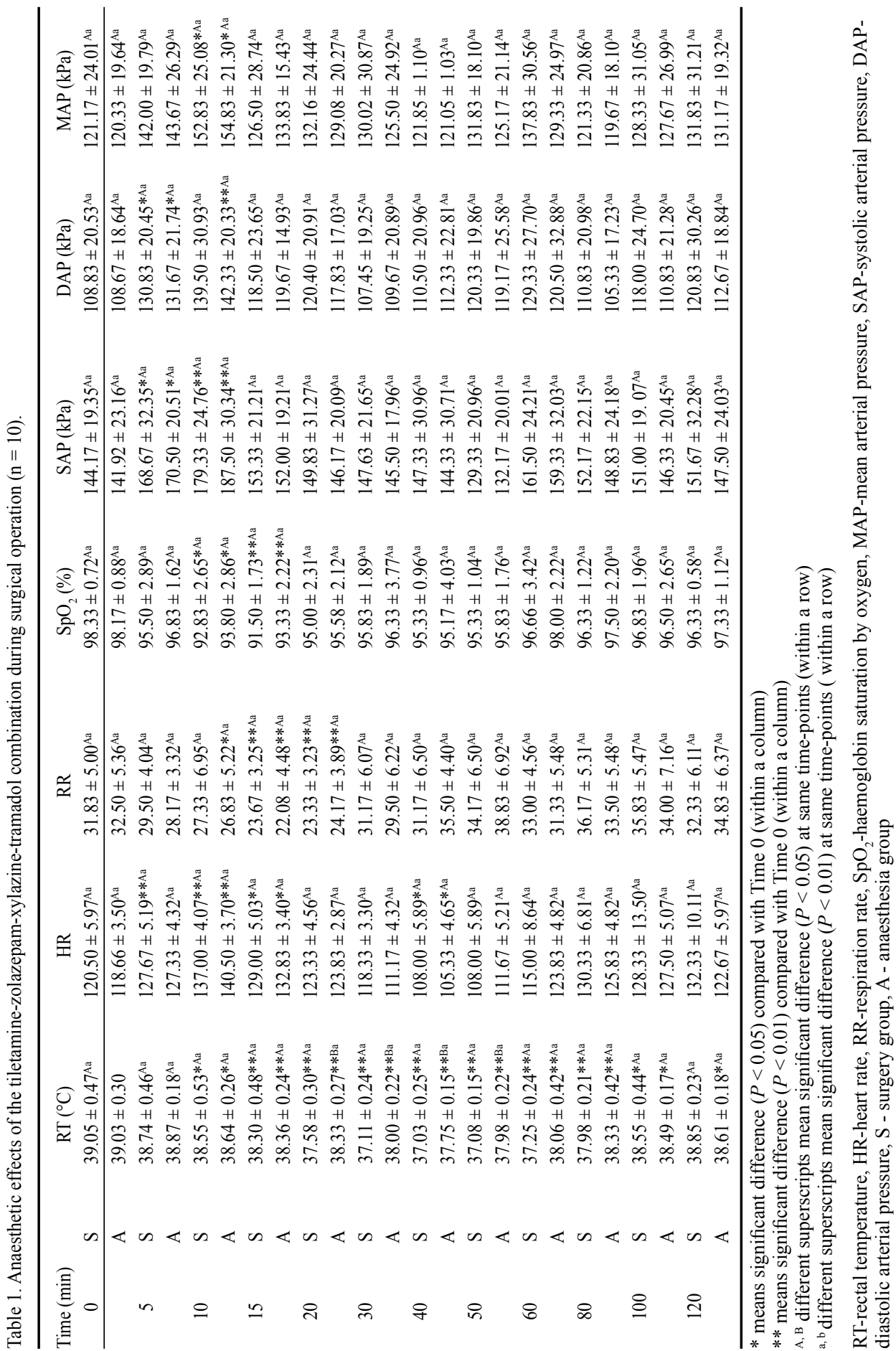




\section{Acknowledgement}

This study was supported by the National Natural Science Foundation of China (Grant No. 31302149 and Grant No. 31001092) and Science and Technology Research Project of Heilongjiang Province Department of Education (Grant No. 12521003). Thanks are due to Dr. Jing Dong for the design and modification of this thesis, she contributed equally to Dr. Lin Li, and should be considered as joint first author.

\section{References}

Alexis MC, Francis JG, Lisa AC, Lindsay WM, Julie KL 2004: Anesthetic and physiologic effects of tiletamine, zolazepam, ketamine, and xylazine combination (TKX) in feral cats undergoing surgical sterilization. J Feline Med Surg 6: 297-303

Bednarski RM, Muir WW, Tracy CH 1989: Effects of tolazoline, doxapram, and RO15-1788 on the depressant action of tiletamine/zolazepam. Vet Med 84: 1016-1022

Degerfeld MM 2005: Personal experiences in the use of Zoletil $®$ for anesthesia of the red-necked wallaby (Macropus rufogriseus). Vet Res Commun 29: 297-300

Doherty TJ 1988: Physiologic effects of alpha 2-adrenergic receptors. J Am Vet Med A 192: 1612-1614

Engelhardt T, Steel E, Johnston G 2003: Tramadol for pain relief in children undergoing tonsillectomy: a comparison with morphine. Paediatr Anaesth 13: 249-252

Hellyer P, Muir W, Hubbell JA 1988: Cardiorespiratory effects of the intravenous administration of tiletaminezolazepam to cats. Vet Surg 17:105-110

James SB, Cook RA, Raphael BL 1999: Immobilization of babirusa (Babyrousa babyrussa) with xylazine and tiletamine/zolazepam and reversal with yohimbine and flumazenil. J Zoo Wildl Med 30: 521-525

Lehmann KA 1997: Tramadol in acute pain. Drugs 53: 25-33

Lemke KA 2004: Perioperative use of selective $\alpha-2$ agonists and antagonists in small animals. Can Vet J 45: 475-480

Lin HC, Thurmon JC, Benson GJ 1993: Telazol - a review of its pharmacology and use in veterinary medicine. J Vet Pharmacol Ther 16: 383-418

Lin Li, Jing Dong, Dezhang Lu, Sheng Jing, Dongqi Lin, Honggang Fan 2012: Effects of leamine zolazepam xylazine tramadol combination on biochemial and haematologial Parmeters in Cats. Bull Vet Inst Pulawy 56: 369-372

Safarinejad MR, Hosseini SY 2006: Safety and efficacy of tramadol in the treatment of idiopathic detrusor overactivity: a double-blind, placebo-controlled, randomized study. Br J Clin Pharmacol 61: 456-463

Shilo Y, Britzi M, Eytan B 2008: Pharmacokinetics of tramadol in horses after intravenous, intramuscular and oral administration. J Vet Pharmacol Ther 31: 60-65

Sousa AB, Santos ACD, Schramm SG 2008: Pharmacokinetics of tramadol and O-desmethyltramadol in goats after intravenous and oral administration. J Vet Pharmacol Ther 31:45-51

Witte JD, Schoenmaekers B, Sessler DL 2001: The analgesic efficacy of tramadol is impaired by concurrent administration of ondansetron. Anesth Analg 92: 1319-1321 